\title{
The non-responsiveness to anti-vascular endothelial growth factor agents in the treatment of neovascularage-related macular degeneration
}

\begin{abstract}
In this editorial, the definition and potential risk factors of the non-responsiveness to the intravitreal applications of anti-vascular endothelial growth factors in the patients with neovascular age-related macular degeneration were summarized. To know the potential risk factors for the non-responsiveness to these agents may prevent unnecessary treatment interventions and unrealistic patient expectations.
\end{abstract}

Keywords: anti-vascular endothelial growth factor, age-related macular degeneration, non-responsiveness, definition, non-responders, potential risk factors
Volume 6 Issue $6-2017$

\author{
Burak Turgut \\ Department of Ophthalmology, Firat University, Turkey \\ Correspondence: Burak Turgut, Associate Professor \\ of Ophthalmology, Firat University, Faculty of Medicine, \\ Department of Ophthalmology, 23 I I 9 Elazig Turkey, \\ Tel +904242333555, Email burakturgut@firat.edu.tr
}

Received: April 27, 2017| Published: April 28, 2017
Abbreviations: Anti-VEGF, anti-vascular endothelial growth factor; n-AMD, neovascular age-related macular degeneration; BVCA, best corrected visual acuity; PED, pigment epithelium detachment

\section{Editorial}

The anti-vascular endothelial growth factor (anti-VEGF) agents including bevacizumab, ranibizumab, and aflibercept have been widely used in the current treatment of neovascular age-related macular degeneration (n-AMD), and they are beneficial in most n-AMD patients..$^{1-5}$ However, some patients cannot respond to the treatment as expected. In recent studies, it has been showed that the incidence for non-responsiveness to bevacizumab in treatment naïve n-AMD ranges between approximately 40 and $50 \%$ while as the percent of non-responsiveness to ranibizumab was between approximately 10$20 \%{ }^{6-9}$ Although there is currently no more evidence in the literature, a recent report demonstrated that the rates of non-responsiveness to aflibercept in the treatment of $\mathrm{n}$ AMD based on best corrected visual acuity (BVCA) and fundus findings were approximately $8 \%$ and $13 \%$, respectively. ${ }^{10}$

The absence of expected response to an anti-VEGF agent has been named in different forms as "incomplete response, poor response, nonresponse, unresponsive, tolerance, tachyphylaxis, rebound, treatment resistant, refractory to anti-VEGF, resistance to anti-VEGF" in current ophthalmology literature. ${ }^{11}$ However, non responsiveness is exactly different from above mentioned most nomenclatures. If the mistaken diagnosis, tachyphylaxis, and complications secondary to the drug's itself or its application were excluded, the non-responsiveness to intravitreal anti-VEGF injection in the treatment of n-AMD is defined as the loss (more than 0.2 or 5 letters) or no change in BVCA compared to baseline with persistent macular hemorrhage in fundus examination, intraretinal or subretinal fluid on optical coherence tomography and the leakage in fluorescein angiography despite to a protocol including 3 or 6 consecutive monthly intravitreal anti-VEGF injections. ${ }^{6-12}$ However, there is no consensus on the defining of the non responsiveness. In the light of the recent reports, it can be considered that the potential risk factors for non-responsiveness to anti-VEGF agent in the treatment of $\mathrm{n}-\mathrm{AMD}$ are an initial lesion with subfoveal fibrosis or atrophy in retina pigment epithelium and photoreceptors, lesion in large size, type 1 choroidal neovascularization, serous pigment epithelium detachment (PED), haemorrhagic PED, fibrovascular PED, polypoidal choroidal vasculopathy, foveal scarring and vitreomacular traction, outer retinal tubulation, cystoid degeneration in outer retina, genetic disposition or an anti-VEGF resistance (Table 1). ${ }^{13-22}$

Table I The possible risk factors for non-responsiveness to anti-VEGF agents in the patients with $n A M D^{6-22}$

\begin{tabular}{l} 
Possible risk factor for non-responsiveness \\
\hline Subfoveal fibrosis/scarring \\
Atrophy in RPE and photoreceptors \\
Large lesion size \\
Type I CNV \\
Serous PED \\
Hemorrhagic PED \\
Fibrovascular/vascular PED \\
PCV \\
VMT \\
ORT \\
Cystoid degeneration in outer retina \\
Genetic disposition \\
Anti-VEGF resistance
\end{tabular}

Table Abbreviations: RPE, retina pigment epithelium; CNV, choroidal neo-vascularization; PED, pigment epithelium detachment; PCV, polypoidal choroidal vasculopathy; VMT, vitreo-macular traction; ORT, outer retinal tubulation;Anti-VEGF, anti-vascular endothelial growth factor

\section{Conclusion}

To know the definition and the potential risk factors of the antiVEGF non responsiveness in the treatment of n-AMD would prevent unnecessary treatment interventions, unrealistic patient expectations, and economic consumption. 


\section{Acknowledgements}

None.

\section{Conflicts of interest}

The authors declare there are no conflicts of interest.

\section{References}

1. Avery RL, Pieramici DJ, Rabena MD, et al. Intravitrealbevacizumab (Avastin) for neovascular age-related macular degeneration. Ophthalmology. 2006;113(3):363-372.

2. Abraham P, Yue H, Wilson L. Randomized, double-masked, sham controlled trial of ranibizumab for neovascular age-related macular degeneration: PIER study year 2. Am J Ophthalmol. 2010;150(3):315-324.

3. Chang TS, Bressler NM, Fine JT, et al. Improved vision-related function after ranibizumab treatment of neovascular age-related macular degeneration: results of a randomized clinical trial. Arch Ophthalmol. 2007;125(11):1460-1469.

4. Heier JS, Brown DM, Chong V, et al. Intravitreal aflibercept (VEGF trap-eye) in wet age-related macular degeneration. Ophthalmology. 2012;119(12):2537-2548

5. Ogura Y, Terasaki H, Gomi F, et al. Efficacy and safety of intravitreal aflibercept injection in wet age-related macular degeneration: outcomes in the Japanese subgroup of the VIEW 2 study. $\mathrm{Br} J$ Ophthalmol. 2015;99(1):92-97.

6. Suzuki M, Nagai N, Izumi-Nagai K, et al. Predictive factors for nonresponse to intravitreal ranibizumab treatment in age-related macular degeneration. Br J Ophthalmol. 2014;98(9):1186-1191.

7. Lux A, Llacer H, Heussen FM, et al. Non-responders to bevacizumab (Avastin) therapy of choroidal neovascular lesions. Br J Ophthalmol. 2007;91(10):1318-1322.

8. Krebs I, Glittenberg C, Ansari-Shahrezaei S, et al. Non-responders to treatment with antagonists of vascular endothelial growth factor in agerelated macular degeneration. Br J Ophthalmol. 2013;97(11):1443-1446.

9. Otsuji T, Nagai Y, Sho K, et al. Initial non-responders to ranibizumab in the treatment of age-related macular degeneration (AMD). Clin Ophthalmol. 2013;7:1487-1490.

10. Nagai N, Suzuki M, Uchida A, et al. Non-responsiveness to intravitrealaflibercept treatment in neovascular age-related macular degeneration: implications of serous pigment epithelial detachment. Sci Rep. 2016;6:29619.
11. Yang S, Zhao J, Sun X. Resistance to anti-VEGF therapy in neovascular age-related macular degeneration: a comprehensive review. Drug Des Devel Ther. 2016;10:1857-1867.

12. Lalwani GA, Rosenfeld PJ, Fung AE, et al. A variable-dosing regimen with intravitrealranibizumab for neovascular age-related macular degeneration: year 2 of the PrONTO Study. Am J Ophthalmol. $2009 ; 148(1): 43-58$.

13. Yamashiro K, Tomita K, Tsujikawa A, et al. Factors associated with the response of age-related macular degeneration to intravitreal ranibizumab treatment. Am J Ophthalmol. 2012;154(1):125-136.

14. Dirani A, Ambresin A, Marchionno L, et al Factors influencing the treatment response of pigment epithelium detachment in age-related macular degeneration. Am J Ophthalmol. 2015;160(4):732-738.

15. Amoaku WM, Chakravarthy U, Gale R, et al. Defining response to antiVEGF therapies in neovascular AMD. Eye. 2015;29(6):721-731.

16. Suzuki M, Nagai N, Shinoda $H$, et al. Distinct responsiveness to intravitreal ranibizumab therapy in polypoidal choroidal vasculopathy with single or multiple polyps. Am J Ophthalmol. 2016;166:52-59.

17. Turgut B, Tanyildizi R. The frequency of the nonresponsiveness to intravitreal injection of the anti-vascular endothelial growth factor agent in neovascular age related macular degeneration. Int J Ophthalmic Pathol. 2013;2:3.

18. Korb C, Zwiener I, Lorenz K, et al. Risk factors of a reduced response to ranibizumab treatment for neovascular age-related macular degeneration-evaluation in a clinical setting. BMC Ophthalmol. 2013;13:84.

19. Rich RM, Rosenfeld PJ, Puliafito CA, et al. Short-term safety and efficacy of intravitreal bevacizumab (Avastin) for neovascular agerelated macular degeneration. Retina. 2016;26(5):495-511.

20. Brantley MA, Fang AM, King JM, et al. Association of complement factor $\mathrm{H}$ and LOC387715 genotypes with response of exudative age-related macular degeneration to intravitreal bevacizumab. Ophthalmology. 2007;114(12):2168-2173.

21. Manoj S. Why does anti VEGF treatment fail in age related macular degeneration (AMD). Kerala Journal of Ophthalmology. 2011;3(3):282-286.

22. Lee AY, Raya AK, Kymes SM, et al. Pharmacogenetics of complement factor $\mathrm{H}(\mathrm{Y} 402 \mathrm{H})$ and treatment of exudative age-related macular degeneration with ranibizumab. Br J Ophthalmol. 2009;93(5):610-613. 